\title{
Diabetes aggravates acute pancreatitis and inhibits pancreas regeneration in mice
}

\author{
D. Zechner • M. Spitzner • A. Bobrowski • N. Knapp • \\ A. Kuhla • B. Vollmar
}

Received: 20 December 2011 / Accepted: 11 January 2012 /Published online: 12 February 2012

(C) Springer-Verlag 2012

\begin{abstract}
Aims/hypothesis It is well established that acute pancreatitis often causes diabetes and that a high blood glucose level associated with pancreatitis is a marker of poor prognosis. The aim of this study was to evaluate if diabetes merely reflects the severity of pancreatitis or whether it can also aggravate the progression of this disease in a vicious circle. Methods Reversible acute oedematous pancreatitis was induced in untreated and streptozotocin-treated diabetic mice by injection of cerulein. Progression of pancreatitis was studied by immunohistochemistry, ELISA and various other enzyme assays. The production of regenerating isletderived $3 \beta$ (REG3 $\beta$ ) was determined by western blot and immunohistochemistry.

Results While cerulein treatment in non-diabetic mice resulted in acute pancreatitis followed by regeneration of the pancreas within 7 days, diabetes aggravated pancreatitis, inhibited the regeneration of the exocrine tissue and led to strong atrophy of the pancreas. The aggravation of pancreatitis by diabetes was characterised by decreased production of the anti-inflammatory protein REG3 $\beta$, increased inflammation, augmented oedema formation and increased cell death during the acute phase of pancreatitis $(p<0.05)$. During the regenerative phase, diabetes augmented inflammation, increased cell death, reduced acinar cell expansion and increased the expansion of duct as well as interstitial cells, resulting in the
\end{abstract}

Electronic supplementary material The online version of this article (doi:10.1007/s00125-012-2479-3) contains peer-reviewed but unedited supplementary material, which is available to authorised users.

D. Zechner · M. Spitzner • A. Bobrowski $\cdot$ N. Knapp · A. Kuhla •

B. Vollmar $(\triangle)$

Institute for Experimental Surgery, University of Rostock,

Schillingallee $69 \mathrm{a}$,

18057 Rostock, Germany

e-mail: brigitte.vollmar@med.uni-rostock.de formation of tubular complexes $(p<0.05)$. Administration of insulin reversed the observed phenotype in diabetic mice.

Conclusions/interpretation Diabetes aggravates acute pancreatitis and suppresses regeneration of the exocrine tissue. Thus, diabetes is not just a concomitant phenomenon of pancreatitis, but can have a fundamental influence on the progression of acute pancreatitis.

Keywords Diabetes - Insulin · Pancreas regeneration · Pancreatitis $\cdot$ REG3 $\beta$

$\begin{array}{ll}\text { Abbreviations } \\ \text { AP } & \text { Acute pancreatitis } \\ \text { CAE } & \text { Chloroacetate esterase } \\ \text { REG3 } \beta & \text { Regenerating islet-derived } 3 \beta \\ \text { STZ } & \text { Streptozotocin } \\ \text { s.c. } & \text { Subcutaneously } \\ \text { TAP } & \text { Trypsinogen-activating peptide }\end{array}$

\section{Introduction}

Acute pancreatitis (AP) is an inflammatory disease of variable severity with an overall mortality rate of $2-3 \%$ [1]. In most cases AP is mild and heals without any further complications. However, $15-20 \%$ of patients develop a severe form of AP, which is characterised by presence of organ failure and/or local pancreatic complications such as the development of a pseudocyst, abscess or parenchymal necrosis [1]. Most clinical centres report mortality rates for severe AP of $5-15 \%$ and some reports even present mortality rates of up to $25 \%[1,2]$.

There is a well-recognised correlation between hyperglycaemia and acute as well as chronic pancreatitis in patients $[3,4]$. For example, temporary hyperglycaemia 
can be observed in up to $50 \%$ of patients with AP, persistent diabetes may affect $1-15 \%$ of patients with AP, and the prevalence of diabetes in chronic pancreatitis varies between $30 \%$ and $83 \%$, depending on the study [5, 6]. This correlation is usually explained by the accepted fact that pancreatitis can cause the development of diabetes mellitus [3]. The investigation into whether diabetes can also cause the aggravation of pancreatitis is less tangible [3]. However, several publications suggest that this cause-and-effect relationship might exist. For example, patients with diabetes have been reported to have a higher risk for pancreatitis [7-10], and hyperglycaemia may predispose patients with acute pancreatitis to systemic organ failure [11]. Blood glucose level is an accurate predictor of outcome in gallstone pancreatitis [12] and an important criterion for the Ranson score, which is used to assess the prognosis of AP [13]. All of these clinical studies support the hypothesis that hyperglycaemia may aggravate pancreatitis. However, these correlative studies cannot prove a causal relationship.

An animal model widely used to study reversible oedematous AP relies on the induction of pancreatic injury with the administration of cerulein, an analogue of the pancreatic secretagogue cholecystokinin. Administration of a supraphysiological dose of cerulein activates trypsinogen within acinar cells, possibly by causing a co-localisation of digestive zymogens with lysosomal enzymes [14, 15]. After the acute phase of pancreatitis, acinar cells respond to the tissue injury by dedifferentiation to immature progenitor-like cells, followed by massive proliferation and redifferentiation into acinar cells. This leads to a recovery of the exocrine tissue within 7 days after AP [16-18]. During the acute phase of pancreatitis the pancreas initiates an acute emergency programme for protecting the parenchyma. As part of this emergency programme, the expression of genes such as $\operatorname{Reg} 3 \beta$ (also known as Pap, PapI, Reg-III and Hip), is strongly upregulated [19]. By using blocking antibodies, antisense oligonucleotides or genetic manipulation of mice, it has been demonstrated that regenerating islet-derived $3 \beta$ (REG3 $\beta$ ) inhibits inflammation and protects the pancreas after ceruleininduced pancreatitis [20-22]. Thus, REG3 $\beta$ is a key factor of the acute emergency programme.

The purpose of this present study was to explore the question of whether diabetes can influence the progression of AP and to analyse which aspects of this disease are affected by diabetes.

\section{Methods}

Induction of diabetes and experimental pancreatitis Male C57BL/6 J mice were purchased from Charles River Laboratories (Sulzfeld, Germany) and were used at 8-12 weeks of age with a body weight of 22-30 g or at 12 months of age with a body weight of 30-35 g. Animals were allowed access to water and standard laboratory chow ad libitum. Diabetes was induced by i.p. injection of $50 \mathrm{mg} / \mathrm{kg}$ streptozotocin (STZ; Sigma-Aldrich, Steinheim, Germany) on 5 consecutive days. Progression of diabetes was monitored with the blood glucose meter Contour (Bayer Vital, Leverkusen, Germany). For insulin treatment, mice received on day 15 one pellet of insulin subcutaneously (s.c.; Linshin Canada, Toronto, $\mathrm{ON}$, Canada; release rate, $0.1 \mathrm{U}$ insulin per day). For treatment with REG3 $\beta$, mice received $100 \mu \mathrm{g} / \mathrm{kg}$ body weight REG3 $\beta$ (Dynabio, Marseille, France) i.v. $2 \mathrm{~h}$ before the first cerulein injection on days 22 and 23. Pancreatitis was induced by administration of eight i.p. injections of $50 \mu \mathrm{g} / \mathrm{kg}$ cerulein (Sigma-Aldrich) at a rate of one every hour over 2 consecutive days. All control mice were sham treated appropriately $(0.9 \%$ wt/vol. $\mathrm{NaCl}$ solution instead of cerulein, $50 \mathrm{mmol} / \mathrm{l}$ sodium citrate $\mathrm{pH} 4.5$ instead of STZ, wounding of the skin with a trocar instead of insulin pellets, PBS instead of REG3 $\beta$ ). At $2 \mathrm{~h}$ before induction of pancreatitis and up to the time point of tissue preservation all mice received drinking water containing $800 \mathrm{mg} / 1 \mathrm{metamizol}$ (Ratiopharm, Ulm, Germany) and $1 \mathrm{~g} / 1$ BrdU (Sigma-Aldrich). BrdU administration had no effect on pancreas atrophy as animals without administration of BrdU had a similar pancreas/body weight ratio on day 30 (STZ + cerulein with BrdU 0.0027 $\pm 0.0012, n=4$; STZ + cerulein without BrdU $0.0026 \pm 0.0012, n=3)$. Animals were anaesthetised with $75 \mathrm{mg} / \mathrm{kg}$ ketamine (bela-pharm, Vechta, Germany) and $5 \mathrm{mg} / \mathrm{kg}$ xylacine (Bayer Health Care, Leverkusen, Germany), before blood samples and organs were taken at the indicated time points. All experiments were approved by the local animal welfare committee and were performed in accordance with the German legislation and the principles of laboratory animal care (NIH publication no. 85-23, revised 1985).

Analysis of plasma Blood samples were taken $2 \mathrm{~h}$ after the last cerulein injection. The activity of lipase and amylase in plasma was analysed using the Cobas c111 spectrophotometer (Roche Diagnostics, Mannheim, Germany). The concentration of the trypsinogen-activating peptide (TAP) in plasma was determined by an ELISA kit for TAP (Uscn Life Science, Wuhan, People's Republic of China).

Histology, quantification of oedema formation, immunohistochemistry and western blots Tissue was preserved $2 \mathrm{~h}$ after the last cerulein injection on days 23 or 30 . The tissue was either frozen for analysis of protein concentrations or fixed in 4\% (wt/vol.) phosphate-buffered formalin for 2-3 days, embedded in paraffin and $4 \mu \mathrm{m}$ sections were cut. Histology was evaluated after staining the sections with haematoxylin and eosin. To evaluate the cellular inflammatory response, naphthol AS-D chloroacetate esterase (CAE) staining was performed on sections. Oedema formation was quantified 
as pancreas wet/dry weight ratio by dividing the weight of the pancreas after drying (at $60^{\circ} \mathrm{C}$ for $48 \mathrm{~h}$ ) by the weight of the native pancreas. Cell proliferation was evaluated by immunohistochemistry using mouse anti-BrdU (clone Bu20a, dilution 1:50) and the Universal LSAB ${ }^{+}$Kit/HRP kit (Dako, Hamburg, Germany). Cell death was analysed using the ApopTag Plus Peroxidase in situ detection kit (Millipore, Eschborn, Germany). Immunohistochemistry for REG3 $\beta$ and insulin was performed using a rat-anti-REG3 $\beta$ antibody (R\&D Systems, Minneapolis, MN, USA; code MAB5110, dilution 1:800) or a guinea pig-anti-insulin antibody (Abcam, Cambridge, UK; ab7842, dilution 1:500). For quantification, at least ten fields (each field with 300 to 650 cells using a $\times 40$ objective) were evaluated per mouse. Acinar cells were identified by large round nuclei, granulated cytoplasm and nonfibroblast-like appearance. Duct cells were identified by a cobblestone-like appearance and direct proximity to a duct. Interstitial cells were identified by interstitial localisation in the exocrine tissue (criterion for exclusion was identification as acinar cell, duct cell or endothelial cell). Western blots were performed by separating $25 \mathrm{mg}$ lysed tissue on $14 \%$ (wt/vol.) SDS gels and transferring the proteins to a polyvinyldifluoride membrane (Immobilon-P; Millipore). After blockade with 5\% (wt/vol.) milk powder, membranes were incubated overnight at $4^{\circ} \mathrm{C}$ with a rat-anti-REG3 $\beta$ antibody (R\&D Systems; code MAB5110, dilution 1:1,000), followed by a secondary peroxidase-linked anti-rat antibody (Santa Cruz Biotechnology, Santa Cruz, USA; code sc3823, dilution 1:10,000). For analysis of $\beta$-actin production, membranes were stripped, blocked by $2.5 \%$ (wt/vol.) BSA and incubated with mouse anti- $\beta$-actin antibody (Sigma-Aldrich, St Louis, MO, USA; code A5441, dilution 1:20,000) followed by peroxidaseconjugated anti-mouse antibody (Sigma-Aldrich [USA]; code A9044, dilution 1:60,000). Protein production was visualised by luminol-enhanced chemiluminescence (ECL plus; GE Healthcare, Munich, Germany) and digitalised with ChemiDoc XRS System (Bio-Rad Laboratories, Munich, Germany). Signals were densitometrically assessed and corrected with the signal intensity of $\beta$-actin (Quantity One; Bio-Rad Laboratories).

Statistics Data are given as means and standard deviations. The significance of data was assessed by SigmaStat 3.5 software (SigmaStat, Jandel Corporation, San Rafael, CA, USA). In cases where the assumption of normality and homogeneity of variance across groups was proven, differences between the groups were calculated using the unpaired Student's $t$ test, including correction of the $\alpha$-error according to the Bonferroni probabilities for repeated analysis. In cases where the assumption of normality or the homogeneity of variance across groups failed, the MannWhitney rank sum test was performed, including correction of the $\alpha$-error according to the Bonferroni probabilities for repeated analysis. The criterion for significance was $p<0.05$.

\section{Results}

Diabetes inhibits the remission of cerulein-induced pancreatitis To test whether diabetes influences the progression of pancreatitis, we induced diabetes in C57BL/6 J mice by i.p. injection of STZ (for experimental procedure see Fig. 1a). Diabetes was characterised by average blood glucose concentrations of $20 \mathrm{mmol} / \mathrm{l}$ on day 22 (Fig. 1b) and a reduction in the number of insulin-producing beta cells in the islets of Langerhans (Fig. 1c, d). STZ-treated mice lost $2.7 \pm 4.3 \%$ of their body weight, whereas non-diabetic mice gained $4.9 \pm 3.2 \%$ body weight by day 22 . We induced AP by repeated i.p. injections of cerulein on days 22 and 23 in diabetic (group: STZ + cerulein) as well as in non-diabetic mice (group: cerulein) and compared them with diabetic (group: STZ) and non-diabetic (group: sham) mice without cerulein exposure.

On day 30 normal morphology of the pancreas was observed in cerulein-, STZ- and sham-treated mice (Fig. 2a [not shown
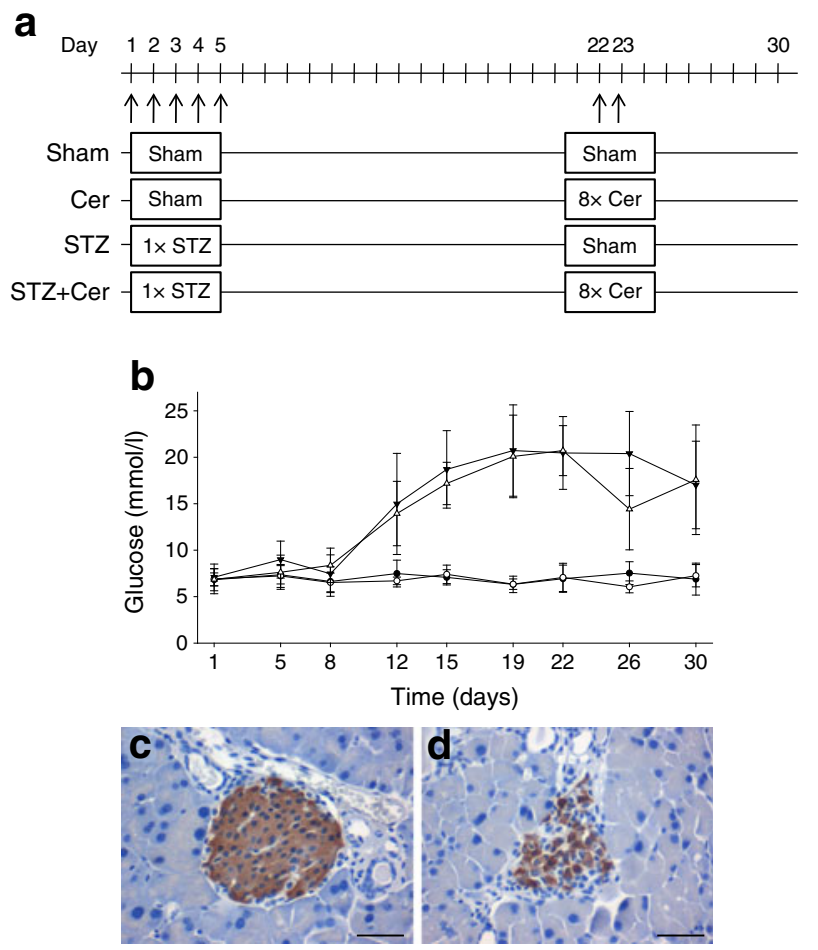

Fig. 1 Experimental protocol, blood glucose levels and insulin production. a Two groups of mice (STZ $n=22 ; \mathrm{STZ}+$ cerulein $n=30)$ were injected i.p. on days $1-5$ with STZ to induce diabetes. Control mice (sham $n=16$; cerulein $n=26$ ) received only the vehicle solution. On days 22 and 23 pancreatitis was induced in two groups of mice ('cerulein' and 'STZ + cerulein') by eight i.p. injection of cerulein at a rate of one every hour, whereas control mice received vehicle solution ('sham' and 'STZ'). b Average blood glucose levels in animals of the four experimental groups. Black diamond, sham; white diamond, cerulein; black triangle, STZ; white triangle, STZ + cerulein. c,d Representative images of antiinsulin immunohistochemistry on day 30 . The brown colour depicts insulin-positive beta cells of sham-treated (c) and STZ-treated (d) mice. Values denote mean \pm SD. Scale bar, $50 \mu \mathrm{m}$. Cer, cerulein 

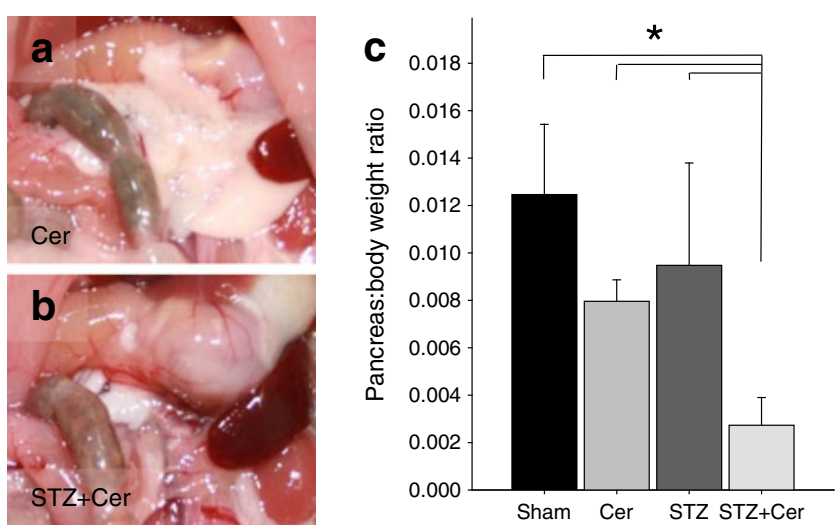

Fig. 2 Pancreas morphology and pancreas/body weight ratio on day 30. Pancreases of a cerulein-treated (a) and a STZ + ceruleintreated (b) mouse. Quantification of the pancreas/body weight ratio revealed profound atrophy of the pancreas in cerulein-treated diabetic mice (c). Values denote mean $\pm \mathrm{SD}$ ( $\operatorname{sham} n=4$; cerulein $n=4$; STZ $n=4$; STZ + cerulein $n=4)$. Significant differences are marked $(*)$. Cer, cerulein

for sham and STZ group]). At that time point, the pancreas had regenerated after cerulein application. However, in mice treated with STZ + cerulein only remnants of the pancreas were visible (Fig. 2b). The pancreas/body weight ratio was reduced in these mice by $78 \%$ when compared with sham-treated animals (Fig. 2c). The reduction in pancreas/body weight ratio of STZ + cerulein-treated mice was significant when compared with the respective values of sham-, cerulein- and STZ-treated animals (Fig. 2c).

Histological analysis of the pancreatic tissue on day 23 revealed the induction of AP in cerulein- as well as in STZ + cerulein-treated animals (Fig. 3a,c). The pancreatic tissue of these mice exhibited marked oedema and the typical feature of tissue-infiltrating inflammatory cells consisting mainly of neutrophils (Fig. 3a,c). The histology of the exocrine tissue was normal in STZ- and sham-treated mice (Fig. 3b). On day 30, no major histological difference was observed between cerulein-, STZ- or sham-treated mice (Fig. 3d,e). However, the pancreases of STZ + cerulein-treated animals

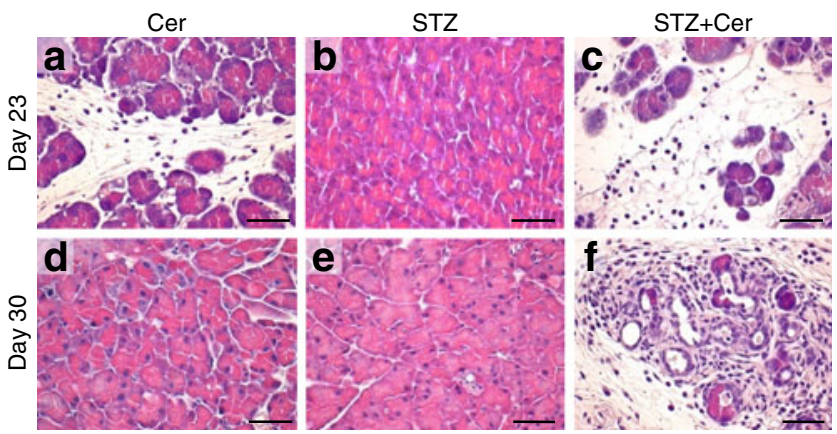

Fig. 3 Haematoxylin and eosin staining of pancreatic tissue specimens. Representative images on day $23(\mathbf{a}-\mathbf{c})$ and day $30(\mathbf{d}-\mathbf{f})$ of mice treated with cerulein (a,d; $n=3)$, STZ (b,e; $n=6)$ and STZ + cerulein $(\mathbf{c}, \mathbf{f} ; n=6)$. Scale bar, $50 \mu \mathrm{m}$. Cer, cerulein were characterised by loss of acinar cells and the appearance of tubular complexes.

Regulation of cell expansion and cell death by diabetes To assess the expansion of specific cell types in the diseased pancreas, BrdU incorporation into replicating nuclei was evaluated at the end of the acute phase on day 23 and at the end of the regenerative phase on day 30 . On day 23 no differences in the number of BrdU-positive acinar or duct cells between the distinct experimental groups were observed (data not shown), but an increase in the number of BrdUpositive interstitial cells was noticed in cerulein- and STZ + cerulein-treated mice $(17.4 \pm 4.2$ and $21.3 \pm 5.7$ cells per field, respectively) when compared with control animals (sham $1.4 \pm 0.7$ cells per field and STZ $2.7 \pm 0.2$ cells per field). On day 30 the number of BrdU-positive interstitial cells was strongly increased in the STZ + cerulein-treated mice compared with sham-, cerulein- and STZ-treated animals (Fig. 4a-d). The number of BrdU-positive acinar cells was significantly elevated in cerulein-treated mice (12-fold) when compared with shamtreated animals (Fig. 4e). However, in STZ + cerulein-treated animals only a modest increase (2.4-fold) in the number of BrdU-positive acinar cells was noticed when compared with STZ-treated animals (Fig. 4e). Interestingly, significantly fewer BrdU-positive acinar cells were also observed in the pancreas of STZ-treated mice when compared with sham-treated animals (Fig. 4e). The number of BrdU-positive duct cells in the pancreas was highly increased in the STZ + cerulein-treated mice when compared with sham-, STZ- and cerulein-treated animals (Fig. 4a,b,f). In order to evaluate the extent of cell death during pancreatitis, we detected double-stranded DNA breaks on histological sections. On days 23 and 30, cells with DNA breaks were primarily observed in cerulein- and STZ + cerulein-treated mice (Fig. 5a-d). At both time points STZ + cerulein-treated animals had a significant increase in the number of cells with DNA breaks when compared with sham-, cerulein- or STZ-treated animals (Fig. 5e,f).

Diabetes affects pancreatic inflammation As ceruleininduced pancreatitis is characterised by infiltration of granulocytes, which strongly express CAE, we evaluated the number of tissue-infiltrating CAE-positive cells in the pancreas. On day 23, almost no CAE-positive cells were observed in STZ- and sham-treated mice, while after treatment with cerulein an increase in the number of CAE-positive infiltrated cells was observed (electronic supplementary material [ESM] Fig. 1a,b; Fig. 6a). An even stronger increase in the number of CAE-positive cells was observed after treatment with STZ + cerulein (ESM Fig. 1c; Fig. 6a). To compare the infiltration of immune cells to the magnitude of tissue oedema, we analysed the pancreas wet/dry weight ratio. In sham-treated mice the wet/dry weight ratio was $3.51 \pm 0.04$. After treatment with cerulein a significant increase in the pancreas wet/dry 

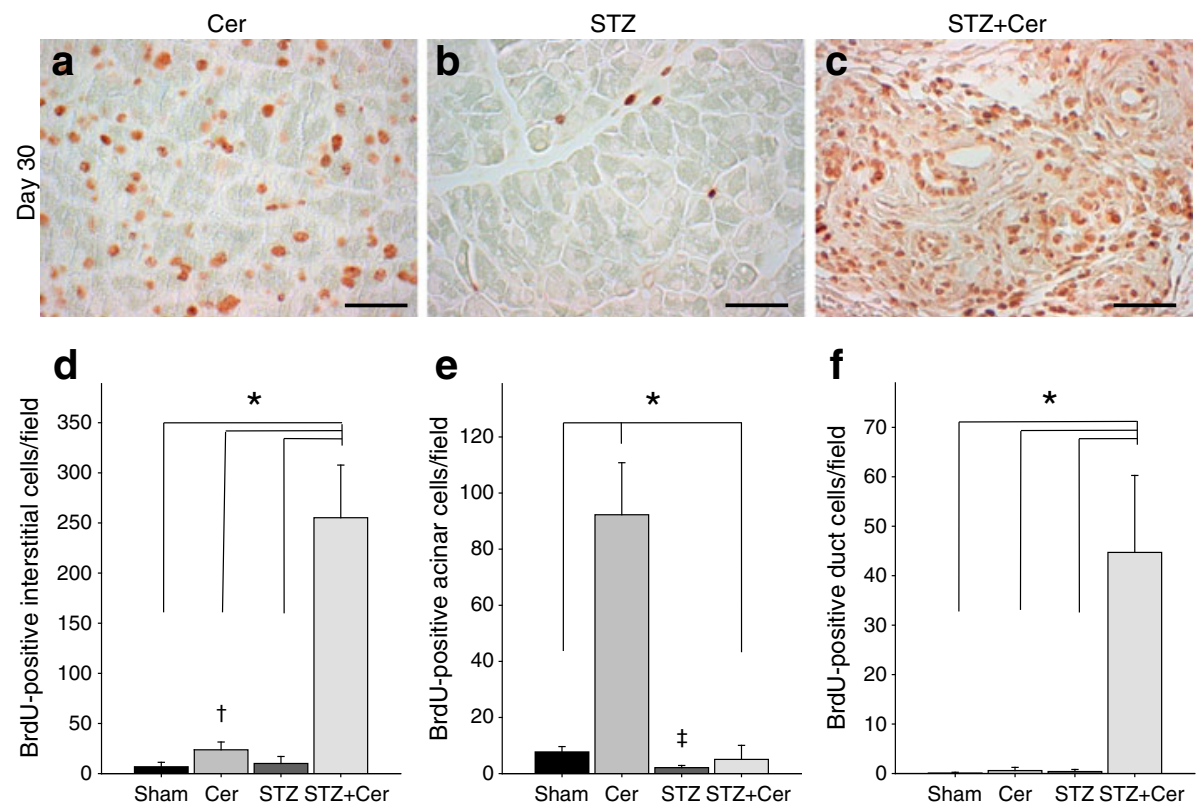

Fig. 4 Detection of proliferative cells by anti-BrdU immunohistochemistry on day 30 . The brown colour depicts BrdU-positive cells from mice treated with cerulein (a), STZ (b) and STZ + cerulein (c). Quantification of BrdU-positive interstitial cells per field (d), of BrdUpositive acinar cells per field on day 30 (e) and of BrdU-positive duct cells per field (f) reveals a cell-type-specific increase in the number of

weight ratio to $4.58 \pm 0.03$ was observed (Fig. $6 \mathrm{~b}$ ). Treatment with STZ + cerulein resulted in a significant rise in the wet/dry weight ratio to $10.31 \pm 0.19$ (Fig. 6b).

On day 30 almost no CAE-positive infiltrating cells were observed in STZ-, cerulein- or sham-treated animals while in STZ + cerulein-treated animals a considerable number of CAE-positive cells were observed (ESM Fig. 1d-f; Fig. 6c).

Activation of trypsinogen and suppression of REG3 $\beta$ production As inappropriate intracellular proteolytic activation of trypsinogen to trypsin has been considered to be the pathological event that initiates pancreatitis [23], we evaluated whether diabetes modulates trypsinogen activation. For this purpose we measured the concentration of TAP in the plasma $2 \mathrm{~h}$ after the last cerulein injection. After treatment with cerulein or STZ + cerulein a twofold increase in the concentration of TAP was observed (Fig. 7a). Importantly, no difference in the concentration of TAP was observed between cerulein- and STZ + cerulein-treated mice (Fig. 7a). We also analysed lipase activity and amylase activity in the plasma. Lipase activity in the plasma was induced eightfold in cerulein-treated mice compared with shamtreated animals and 29-fold in STZ + cerulein-treated mice compared with STZ-treated animals (Fig. 7b). Similarly, the activity of amylase was induced sixfold in cerulein-treated mice compared with sham-treated animals and 13-fold in STZ + cerulein-treated mice compared with STZ-treated animals (Fig. 7c).
BrdU-positive cells in diabetic mice after induction of pancreatitis. Values denote mean $\pm \mathrm{SD}$ ( sham $n=7$; cerulein $n=7$; STZ $n=9$; STZ + Cer $n=8$ ). Symbols denote significant differences between the indicated groups $(*)$, between cerulein- and sham-treated mice $(\dagger)$ or between STZand sham-treated mice ( $\$$ ). Scale bar, $50 \mu \mathrm{m}$. Cer, cerulein

In order to evaluate, whether the production of REG3 $\beta$, an important protein induced by the emergency programme during pancreatitis, is altered in diabetic mice, we analysed the production of this protein by immunohistochemistry. REG3 $\beta$ production was not detected in the acinar cells of sham- or STZ-treated mice, but was produced in abundance $2 \mathrm{~h}$ after the last cerulein treatment in diabetic as well as non-diabetic mice (Fig. 7d-f; not shown for sham group). In order to quantify REG3 $\beta$, we analysed relative protein concentrations by western blot. After cerulein administration STZ-treated animals showed a significantly lowered level of REG3 $\beta$ production when compared with cerulein-treated non-diabetic animals (Fig. $7 \mathrm{~g}, \mathrm{~h}$ ). We also evaluated whether administration of REG3 $\beta$ can inhibit the inflammation during pancreatitis. On day 23, we observed a small $5.3 \%$ decrease (not significant) in the number of CAE-positive cells in STZ+ REG3 $\beta+$ cerulein-treated mice when compared with STZ + cerulein-treated animals (data not shown).

Progression of pancreatitis in insulin-treated and aged mice In order to evaluate whether the observed phenotype is reversible by treating diabetes with insulin, we implanted insulin-containing pellets s.c. in STZ-treated mice on day 15 . Insulin treatment reduced the glucose concentration by day 22 (Fig. 8a). After induction of pancreatitis we observed on day 30 that STZ + insulin + cerulein-treated mice had an increased pancreas/body weight ratio, reduced cell death, reduced expansion of interstitial cells as well as 

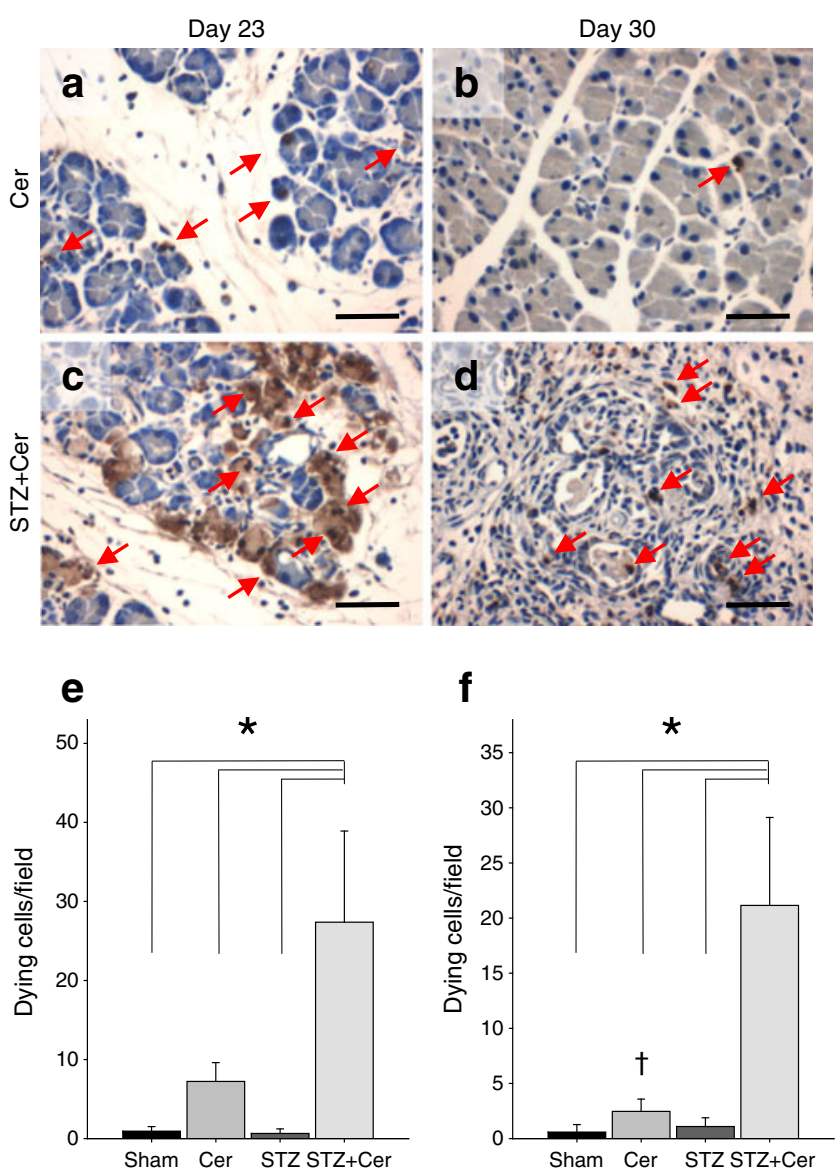

Fig. 5 Detection of cell death. The brown colour depicts cells with DNA double-strand breaks after cerulein $(\mathbf{a}, \mathbf{b})$ and STZ + cerulein $(\mathbf{c}, \mathbf{d})$ treatment on day $23(\mathbf{a}, \mathbf{c})$ and day $30(\mathbf{b}, \mathbf{d})$. Quantification of the number of cells with DNA double-strand breaks on day 23 (e) and day 30 (f) reveals increased cell death after STZ + cerulein treatment. Values denote mean $\pm \mathrm{SD}$ (for day 23, sham $n=4$, cerulein $n=4, \operatorname{STZ} n=4$, STZ + cerulein $n=6$; for day 30 , sham $n=5$, cerulein $n=7$, STZ $n=5$, STZ + cerulein $n=8$ ). Symbols denote significant differences between the indicated groups $(*)$, or between cerulein- and shamtreated mice $(\dagger)$. Scale bar, $50 \mu \mathrm{m}$. Cer, cerulein duct cells and enhanced expansion of acinar cells (Fig. 8b-f) when compared with STZ + cerulein-treated mice. In order to evaluate whether diabetes has a similar effect in aged mice, we compared the pancreases of STZ- and STZ + cerulein-treated 12-month-old mice. Also in aged mice, diabetes reduced acinar cell, but increased interstitial cell as well as duct cell expansion, increased the number of CAE-positive cells, reduced the pancreas/body weight ratio and increased cell death on day 30 (ESM Fig. 2a-f).

\section{Discussion}

In order to test the hypothesis that diabetes is not only a concomitant phenomenon of AP, but can also aggravate this disease, we compared the progression of pancreatitis after induction of pancreatitis in non-diabetic and diabetic mice. The following observations documented that experimental diabetes caused an aggravation of cerulein-induced AP. Compared with non-diabetic mice, diabetic mice displayed: (1) an enhanced and extended inflammatory response; (2) increased local oedema formation; (3) enhanced cell death; (4) reduced acinar cell expansion but increased duct cell as well as interstitial cell expansion; (5) reduced production of REG3 $\beta$; and (6) increased activity of lipase and amylase in blood plasma. The increased cell death and inhibited regeneration of acinar cells resulted in almost complete atrophy of the pancreas. Administration of insulin lowered the blood glucose concentration and inhibited the observed aggravation of pancreatitis by diabetes. This suggests that the diabetic metabolic state rather than non-specific cytotoxicity of administered compounds aggravates pancreatitis. The observed protective effect of insulin might also explain why evolutionary pressure caused the dispersion of the endocrine islets of Langerhans within a predominantly exocrine organ [24]. This so-called islet-acinar axis might not only be

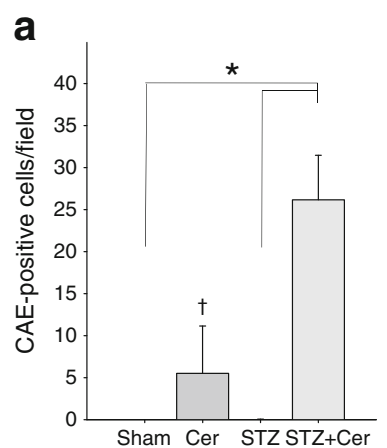

Fig. 6 Quantification of immune cell infiltrates and oedema formation. On day 23 the number of CAE-positive immune cells per field (a) and the pancreas wet/dry weight ratio (b) is increased in cerulein- and STZ + cerulein-treated mice. On day 30 the number of CAE-positive immune cells per field is increased only in diabetic mice after pancreatitis (c).
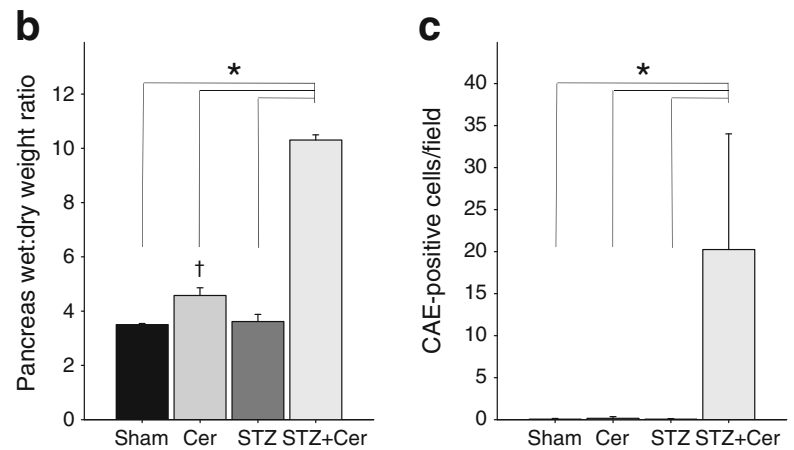

Values denote mean $\pm \mathrm{SD}$ (for day 23 , sham $n=5$, cerulein $n=8, \operatorname{STZ} n=5$, STZ + cerulein $n=8$; for day 30 , sham $n=7$, cerulein $n=7, \operatorname{STZ} n=9, \mathrm{STZ}+$ cerulein $n=8$ ). Symbols denote significant differences between STZ + cerulein-treated mice in comparison with other groups $(*)$, or between cerulein- and sham-treated mice $(\dagger)$. Cer, cerulein 
Fig. 7 Quantification of TAP, lipase, amylase and REG3 $\beta$ production. The concentration of TAP in plasma is increased in mice treated with cerulein $(n=10)$ and STZ + cerulein $(n=15)$ when compared with animals treated with sham $(n=10)$ or STZ $(n=14)(\mathbf{a})$. The activity of lipase (b) and amylase (c) is increased in $\mathrm{STZ}+$ cerulein-treated mice $(n=14)$ compared with sham$(n=11)$, cerulein- $(n=11)$ or STZ-treated animals $(n=13)$. d-f Anti-REG3 $\beta$ immunohistochemistry of mice treated with cerulein $(\mathbf{d} ; n=3), \mathrm{STZ}$ (e; $n=3)$ and STZ + cerulein (f; $n=3)$. Scale bar, $100 \mu \mathrm{m}$. $\mathbf{g , h}$ Representative western blot of REG3 $\beta$ and $\beta$-actin (g) and quantification of REG3 $\beta$ production (sham $n=5$, cerulein $n=12, \operatorname{STZ} n=7, \mathrm{STZ}+\mathrm{Cer}$ $n=14)$ relative to $\beta$-actin (h). Values denote mean \pm SD. Symbols denote significant differences between the indicated groups $(*)$, or between cerulein- and shamtreated mice $(\dagger)$. Cer, cerulein

Fig. 8 Influence of insulin administration on pancreatitis induced atrophy. Insulin administration reduces blood glucose concentration (a) by day 22. Comparison of STZ + cerulein-treated $(n=5)$ with $\mathrm{STZ}+$ insulin + cerulein-treated $(n=6)$ mice on day 30 demonstrates that insulin increases pancreas/body weight ratio (b), reduces cell death (c), reduces the number of BrdU-positive interstitial cells (d), increases the number of BrdU-positive acinar cells (e) and decreases the number of BrdU-positive duct cells (f). Values denote mean \pm SD. Significant differences are marked $(*)$. Cer, cerulein; Ins, insulin
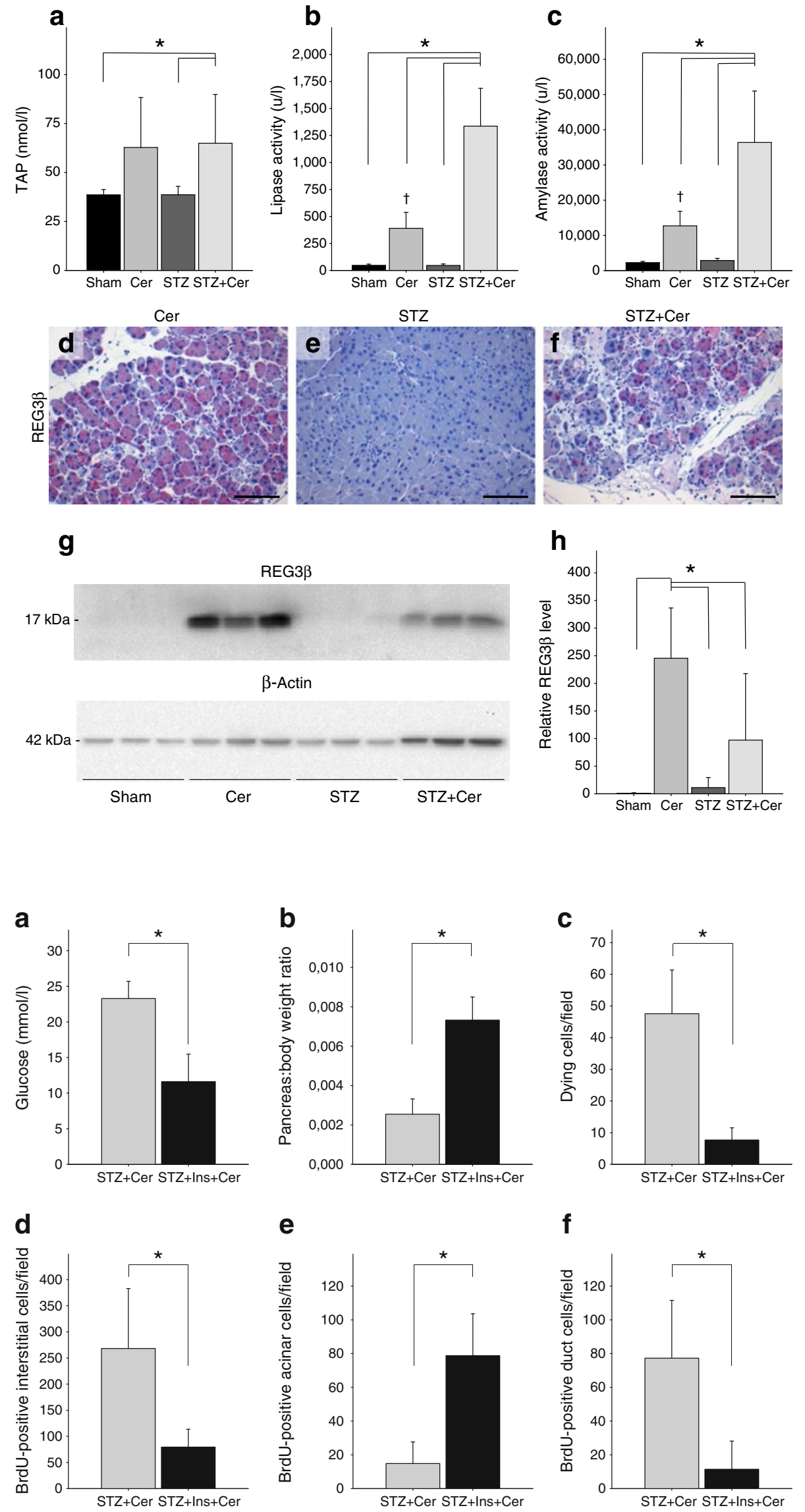
relevant for normal physiology of exocrine tissue, but might be especially relevant for distinct pathologies such as pancreatitis.

AP is thought to be caused by the intracellular activation of trypsinogen [23]. However, in our study diabetes had no influence on the concentration of TAP in the plasma of mice $2 \mathrm{~h}$ after the last cerulein injection, suggesting that diabetes might influence downstream mechanisms of pancreatitis rather than the activation of trypsinogen. One of these downstream mechanisms might be the induction of the acute emergency programme. REG3 $\beta$ is part of the acute emergency programme for protecting the pancreas and has been shown to inhibit inflammation during pancreatitis [20-22]. We demonstrate that REG3 $\beta$ production is significantly suppressed in diabetic mice during pancreatitis. This finding is surprising, as REG3 $\beta$ production usually correlates with the severity of pancreatitis in animal models as well as in patients $[25,26]$. The suppression of REG3 $\beta$ production suggests that the inhibitory mechanism of diabetes on REG3 $\beta$ production overwhelms the stimulatory mechanisms of aggravated pancreatitis. However, we observed only a small non-significant repression of inflammation after administration of REG3 $\beta$. These data suggest that diabetes significantly alters the induction of the emergency programme during pancreatitis, but that several additional factors in addition to reduced REG3 $\beta$ production might contribute to the aggravation of pancreatitis in diabetic mice. We did not observe an obvious increase in REG3 $\beta$ protein in STZ-treated mice when compared with sham-treated animals, whereas other groups could demonstrate an increase in Reg $3 \beta$ RNA level in diabetic animals $[27,28]$. These seemingly conflicting results could be explained by assuming that the detection limit for $\operatorname{Reg} 3 \beta$ RNA might be lower than the detection limit for REG3 $\beta$ protein or that results from RNA analysis do not always correlate with the actual protein content.

Differences between diabetic and non-diabetic mice were first observed $2 \mathrm{~h}$ after the last cerulein injection and included reduced production of REG3 $\beta$, increased activity of lipase and amylase in blood plasma, enhanced inflammatory response, increased local oedema formation and increased cell death. Other differences such as reduced acinar cell expansion were observed on day 30 , but not on day 23 . This observation is consistent with data describing that the main cell expansion of acinar cells occurs during the regenerative, but not the acute, phase of pancreatitis [16, 17]. Interestingly, a significant reduction in BrdU-positive acinar cells was observed in diabetic mice even in the absence of pancreatitis when comparing diabetic mice with sham-treated animals. These data suggest that diabetes might directly inhibit the expansion of acinar cells independent of pancreatitis. In addition, we observed that STZ + cerulein treatment influenced cell expansion in a celltype-specific manner. Diabetes inhibited the expansion of acinar cells, whereas it induced the expansion of interstitial cells and duct cells after pancreatitis. This stimulatory effect on the expansion of these cells might, however, constitute the response of the tissue to increased tissue damage in diabetic mice.

The distinct proliferative behaviour of acinar and duct cells in response to diabetes may lead to the observed formation of tubular complexes in diabetic mice. Though the significance of tubular complexes is controversial, they have been proposed to be early cancer precursors $[29,30]$ and are formed by transdifferentiation of acinar cells to duct cells, as well as increased proliferation of duct cells [31]. Possibly, the increased formation of tubular complexes in diabetic mice after pancreatitis may provide an explanation for the observation that diabetes and chronic pancreatitis are major risk factors for pancreatic cancer [32-35].

Up to $50 \%$ of patients with AP have an elevated blood sugar level $[3,5,6]$. In these patients hyperglycaemia may be caused by decreased insulin secretion, parenteral nutritional therapy, increased gluconeogenesis and decreased glucose use [3]. These patients, however, are also susceptible to hypoglycaemia, resulting in conservative insulin therapy at intensive care units [3]. During the last 10 years an intensified insulin therapy for critically ill patients has been a major issue of discussion. An intensified insulin therapy has been found to be of benefit or of no benefit to patients, depending on the clinical study [36-38]. Possibly this discrepancy may be resolved by the assumption that an intensified insulin therapy is beneficial to certain subsets of patients, whereas in other subsets of critically ill patients the risk of hypoglycaemia will outweigh beneficial effects. Thus, it is essential to understand whether diabetes can aggravate distinct diseases such as AP or whether diabetes is just a concomitant phenomenon of specific diseases. Additional preclinical as well as clinical studies might, therefore, be expedient to provide evidence for the decision on whether repetitive blood glucose measurements or technological advances such as continuous glucose monitoring systems followed by precise blood glucose control may improve the survival of patients with severe AP.

Acknowledgements We thank B. Blendow, D. Butzlaff, D. Frenz and M. Nerowski (Institute for Experimental Surgery, University of Rostock) for excellent technical assistance.

Funding The study was supported by a grant from the FORUN programme of the University of Rostock (project. 889017) and a stipend to A. Bobrowski by the Studienstiftung des deutschen Volkes e.V.

Contribution statement DZ was responsible for the conception of the study, analysis and interpretation of data, drafting the article and revising the article critically. $\mathrm{MS}, \mathrm{AB}, \mathrm{NK}$ and $\mathrm{AK}$ were responsible for analysis and interpretation of data and revising the article critically. BV was responsible for conception of the study and revising the manuscript critically. All authors approved the final version of the manuscript to be published. 
Duality of interest The authors declare that there is no duality of interest associated with the manuscript.

\section{References}

1. Forsmark CE, Baillie J (2007) AGA Institute technical review on acute pancreatitis. Gastroenterology 132:2022-2044

2. Gravante G, Garcea G, Ong SL et al (2009) Prediction of mortality in acute pancreatitis: a systematic review of the published evidence. Pancreatology 9:601-614

3. Czako L, Hegyi P, Rakonczay Z Jr, Wittmann T, Otsuki M (2009) Interactions between the endocrine and exocrine pancreas and their clinical relevance. Pancreatology 9:351-359

4. Angelopoulos N, Dervenis C, Goula A et al (2005) Endocrine pancreatic insufficiency in chronic pancreatitis. Pancreatology 5:122-131

5. Shenoy SD, Cody D, Rickett AB, Swift PG (2004) Acute pancreatitis and its association with diabetes mellitus in children. J Pediatr Endocrinol Metab 17:1667-1670

6. Raman VS, Loar RW, Renukuntla VS et al (2011) Hyperglycemia and diabetes mellitus in children with pancreatitis. J Pediatr 158:612-616

7. Girman CJ, Kou TD, Cai B et al (2010) Patients with type 2 diabetes mellitus have higher risk for acute pancreatitis compared with those without diabetes. Diabetes Obes Metab 12:766-771

8. Noel RA, Braun DK, Patterson RE, Bloomgren GL (2009) Increased risk of acute pancreatitis and biliary disease observed in patients with type 2 diabetes: a retrospective cohort study. Diabetes Care 32:834-838

9. Renner IG, Savage WT 3rd, Pantoja JL, Renner VJ (1985) Death due to acute pancreatitis. A retrospective analysis of 405 autopsy cases. Dig Dis Sci 30:1005-1018

10. Seicean A, Tantau M, Grigorescu M, Mocan T, Seicean R, Pop T (2006) Mortality risk factors in chronic pancreatitis. J Gastrointestin Liver Dis 15:21-26

11. Mentula P, Kylanpaa ML, Kemppainen E, Puolakkainen P (2008) Obesity correlates with early hyperglycemia in patients with acute pancreatitis who developed organ failure. Pancreas 36:e21-e25

12. Rajaratnam SG, Martin IG (2006) Admission serum glucose level: an accurate predictor of outcome in gallstone pancreatitis. Pancreas 33:27-30

13. Ranson JH, Rifkind KM, Roses DF, Fink SD, Eng K, Spencer FC (1974) Prognostic signs and the role of operative management in acute pancreatitis. Surg Gynecol Obstet 139:69-81

14. Frossard JL, Pastor CM (2002) Experimental acute pancreatitis: new insights into the pathophysiology. Front Biosci 7:d275-d287

15. Halangk W, Lerch MM, Brandt-Nedelev B et al (2000) Role of cathepsin B in intracellular trypsinogen activation and the onset of acute pancreatitis. J Clin Invest 106:773-781

16. Fendrich V, Esni F, Garay MV et al (2008) Hedgehog signaling is required for effective regeneration of exocrine pancreas. Gastroenterology 135:621-631

17. Jensen JN, Cameron E, Garay MV, Starkey TW, Gianani R, Jensen J (2005) Recapitulation of elements of embryonic development in adult mouse pancreatic regeneration. Gastroenterology 128:728-741

18. Siveke JT, Lubeseder-Martellato C, Lee M et al (2008) Notch signaling is required for exocrine regeneration after acute pancreatitis. Gastroenterology 134:544-555

19. Closa D, Motoo Y, Iovanna JL (2007) Pancreatitis-associated protein: from a lectin to an anti-inflammatory cytokine. World $\mathrm{J}$ Gastroenterol 13:170-174
20. Vasseur S, Folch-Puy E, Hlouschek V et al (2004) p8 improves pancreatic response to acute pancreatitis by enhancing the expression of the anti-inflammatory protein pancreatitis-associated protein I. J Biol Chem 279:7199-7207

21. Zhang H, Kandil E, Lin YY, Levi G, Zenilman ME (2004) Targeted inhibition of gene expression of pancreatitis-associated proteins exacerbates the severity of acute pancreatitis in rats. Scand J Gastroenterol 39:870-881

22. Gironella M, Folch-Puy E, LeGoffic A et al (2007) Experimental acute pancreatitis in PAP/HIP knock-out mice. Gut 56:1091-1097

23. Hirota M, Ohmuraya M, Baba H (2006) The role of trypsin, trypsin inhibitor, and trypsin receptor in the onset and aggravation of pancreatitis. J Gastroenterol 41:832-836

24. Williams JA, Goldfine ID (1985) The insulin-pancreatic acinar axis. Diabetes 34:980-986

25. Keim V, Willemer S, Iovanna JL, Adler G, Dagorn JC (1994) Rat pancreatitis-associated protein is expressed in relation to severity of experimental pancreatitis. Pancreas 9:606-612

26. Iovanna JL, Keim V, Nordback I et al (1994) Serum levels of pancreatitis-associated protein as indicators of the course of acute pancreatitis. Multicentric study group on acute pancreatitis. Gastroenterology 106:728-734

27. Baeza N, Sanchez D, Christa L, Guy-Crotte O, Vialettes B, Figarella C (2001) Pancreatitis-associated protein (HIP/PAP) gene expression is upregulated in NOD mice pancreas and localized in exocrine tissue during diabetes. Digestion 64:233-239

28. Lu Y, Ponton A, Okamoto H, Takasawa S, Herrera PL, Liu JL (2006) Activation of the Reg family genes by pancreaticspecific IGF-I gene deficiency and after streptozotocin-induced diabetes in mouse pancreas. Am J Physiol Endocrinol Metab 291: E50-E58

29. Wagner M, Luhrs H, Kloppel G, Adler G, Schmid RM (1998) Malignant transformation of duct-like cells originating from acini in transforming growth factor transgenic mice. Gastroenterology 115:1254-1262

30. Bockman DE, Guo J, Buchler P, Muller MW, Bergmann F, Friess H (2003) Origin and development of the precursor lesions in experimental pancreatic cancer in rats. Lab Invest 83:853-859

31. Strobel O, Dor Y, Alsina J et al (2007) In vivo lineage tracing defines the role of acinar-to-ductal transdifferentiation in inflammatory ductal metaplasia. Gastroenterology 133:1999-2009

32. Maisonneuve P, Lowenfels AB (2010) Epidemiology of pancreatic cancer: an update. Dig Dis 28:645-656

33. Luo J, Iwasaki M, Inoue M et al (2007) Body mass index, physical activity and the risk of pancreatic cancer in relation to smoking status and history of diabetes: a large-scale population-based cohort study in Japan-the JPHC study. Cancer Causes Control 18:603-612

34. Hassan MM, Bondy ML, Wolff RA et al (2007) Risk factors for pancreatic cancer: case-control study. Am J Gastroenterol 102:2696-2707

35. Stevens RJ, Roddam AW, Beral V (2007) Pancreatic cancer in type 1 and young-onset diabetes: systematic review and meta-analysis. Br J Cancer 96:507-509

36. van den Berghe G, Wouters P, Weekers F et al (2001) Intensive insulin therapy in the critically ill patients. N Engl J Med 345:1359-1367

37. Finfer S, Chittock DR, Su SY et al (2009) Intensive versus conventional glucose control in critically ill patients. N Engl J Med 360:1283-1297

38. Kovalaske MA, Gandhi GY (2009) Glycemic control in the medical intensive care unit. J Diabetes Sci Technol 3:1330-1341 